\author{
Joanna Brzezińska \\ Université de Wrocław \\ ORCID : 0000-0002-5030-3363 \\ joanna.brzezinska@uwr.edu
}

\title{
La criminalité féminine au Canada selon les statistiques*
}

\author{
Przestępczość kobiet w Kanadzie z perspektywy statystyk
}

\author{
RÉSUMÉ
}

Le but de cette étude était de caractériser le phénomène de la criminalité féminine au Canada du point de vue de l'analyse des données statistiques. A cette fin, des résumés ont été présenté en tenant compte de la fréquence du phénomène étudié en termes de trois périodes de recherche: 1975-1981, 1984-1994 et 1999-2009. Il a été établi que les auteurs canadiens exécutent des catégories établies de crimes (crimes contre les biens, crimes ayant recours à la violence) dont la fréquence a changé au cours des périodes examinées. Dans le cadre de la recherche menée, le phénomène d'une augmentation de la fréquence d'infractions commises par des femmes est particulièrement préoccupant. Ce fait indique l'augmentation de la pathologisation de leurs comportements. En outre, au cours de la dernière des périodes de recherche (1999-2009), on a également observé de nouvelles tendances dans le domaine de l'activité criminogène des femmes : les infractions routières et les infractions liées à la drogue et aux stupéfiants. Toutefois, leur degré n'a pas été élevé.

Mots-clés : criminalité féminine ; activité criminogène des femmes au Canada

\section{INTRODUCTION}

La première observation que l'on retrouve dans les délibérations de criminologues canadiens dans le domaine de la criminalité féminine concerne, comme chez les chercheurs européens ${ }^{1}$, la constatation que les femmes représentent une

* Cet article est le résultat d'une recherche financée dans le cadre du projet financé par le Centre national des sciences. Le chef de projet est dr J. Brzezińska (décision n DEC 2015/19/B/HS5/03042).

1 Voir J. Błachut, Niektóre koncepcje kryminologiczne a problem przestępczości kobiet, „Archiwum Kryminologii” 1989, t. 16 ; K. Biel, Przestępczość dziewcząt. Rodzaje i uwarunkowania, 
population des suspectes dont le nombre est peu élevé, toujours inférieur à celle des hommes ${ }^{2}$. Selon le Centre canadien de la statistique juridique, les femmes ont toujours été une sorte de minorité criminelle au Canada ${ }^{3}$, et il en est de même maintenant $t^{4}$. Cette situation est influencée par une diversité des contextes ${ }^{5}$ : biologiques, sociologiques ou économiques, qui déterminent la forme et la nature du comportement féminin ${ }^{6}$. Afin de déterminer correctement les propriétés de la population des suspectes canadiennes, il est nécessaire de caractériser les crimes commis par elles, en les séparant complètement d'infractions des suspects? ${ }^{7}$. De plus, d'après les observations des Canadiennes qui sont entrées en conflit avec la ${ }_{10}{ }^{8}$, il résulte également que les crimes qu'elles commettent montrent un niveau de nocivité sociale inférieur aux infractions caractéristiques des hommes ${ }^{9}$. Les femmes restent généralement concentrées sur certaines catégories d'activités illégales, parmi lesquelles prédominent les crimes contre les biens (vols et cambriolages, fraudes), ou contre la décence (insultes) ${ }^{10}$, et ont donc tendance à se spécialiser dans certaines

Kraków 2009; Z. Majchrzyk, Kiedy kobieta zabija, Warszawa 2009; M. Grzyb, E. Habzda-Siwek, Pteć a przestępczość. O problemie dysproporcji płci, „Archiwum Kryminologii” 2013, t. 23 ; M. Cabalski, Przemoc stosowana przez kobiety, Kraków 2017.

2 M.-A. Bertrand, La femme et le crime, Montréal 1979; R. Collette-Carrière, L. Langelier-Biron, Du côté des filles et des femmes, leur délinquance, leur criminalité, « Les femmes et la justice pénale » 1983, nº 2, DOI : https://doi.org/10.7202/017179ar, p. 2 ; R. Kong, K. AuCoin, R. Kong, K. AuCoin, Les contrevenantes au Canada, « Juristat» 2008, n 1, p. 27-28 ; M. Wallace, J. Turner, A. Matarazzo, C. Babyak, La mesure de la criminalité au Canada : présentation de l'indice de gravité de la criminalité et des améliorations au Programme de déclaration uniforme de la criminalité, Ottawa 2009 ; D. Dhamen, Criminalité, femmes et autochtones, http://web.umoncton.ca/umcm-sitesgr/ robinsg/adpu6055h09/edd.pdf [accès : 10.02.2019], p. 13.

3 Voir C. Collectif, L'Histoire des femmes au Québec depuis quatre siècles, Montréal 1992.

4 B. Myles, Rapport de Statistique Canada - Les crimes avec violence en hausse chez les femmes, 2008, www.ledevoir.com/societe/175671/rapport-de-statistique-canada-les-crimes-avecviolence-en-hausse-chez-les-femmes [accès : 10.02.2019].

5 S. Frigon, Corps, féminité et dangerosité : de la production de " corps dociles » en criminologie, [dans :] S. Frigon, M. Kerisit (dir.), Du corps des femmes : contrôles, surveillances et résistances, Ottawa 2000, p. 134-145.

6 N. Bourgoin, Les chiffres du crime. Statistiques criminelles et contrôle social (France, 18252006), Paris 2008, p. 50 et ss.

7 R. Kong, K. AuCoin, op. cit., p. 1.

8 Voir K. Hannah-Moffat, Punishment in Disguise: Penal Governance and Federal Imprisonment of Women in Canada, Toronto 2001 ; S. Frigon, Transformations de la philosophie et gestion pénale des femmes justiciables au Canada (1990-2000) : trois cas de figure, "Revue Femmes et Droit » 2006, p. 73 et ss.

9 La criminalité chez les femmes au Québec et au Canada, www.alterjustice.org/u9/criminalite_femmes.html [accès : 10.02.2019], p. 1; R. Fitzgerald, op. cit.

10 M. Dauvergne, Statistiques sur les tribunaux de juridiction criminelle pour adultes au Canada 2010-2011, 2012, www.statcan.gc.ca/pub/85-002-x/2012001/article/11646-fra.pdf [accès : 10.02.2019]. 
catégories de comportements illégaux ${ }^{11}$. Cependant, leurs contextes et circonstances restent très différentes de celles réalisées par des suspects ${ }^{12}$.

En regardant actuellement le phénomène de la criminalité féminine, il est impossible de ne pas avoir l'impression que le comportement du sexe féminin au niveau social est évalué d'une manière très extrême. Au Canada, il y a également un stéréotype bien établi de la soi-disante « bonne madone » et " mauvaise suspecte » qui introduit une démarcation simplifiée, excluant d'autres nuances d'activité entre ces attitudes féminines ${ }^{13}$. Dans ce contexte, il convient de citer le point de vue de Lloyd, qui a souligné que :

Lorsque les femmes commettent des crimes violents, elles sont considérées comme des personnes qui enfreignent deux catégories de droits : le droit interne qui interdit la violence et le droit naturel, plus fondamental, selon lequel les femmes manifestent généralement une attitude passive et ne sont pas agressives ${ }^{14}$.

Une femme est présentée comme un individu purement diabolique ou seulement bon, ne laissant aucun espace de liberté pour d'autres comportements, pas nécessairement extrêmes, qu'elle présente habituellement ${ }^{15}$.

Le but de cette étude est de comparer les commentaires ci-dessus avec des données statistiques illustrant la fréquence criminelle des crimes féminins au Canada dans l'optique de trois périodes de recherche : 1975-1981, 1984-1994 et 1999-2009. Il semble nécessaire d'analyser et d'établir les tendances dominantes dans la criminalité des suspectes dans la zone indiquée.

\section{LA CRIMINALITE FEMININE AU CANADA DANS LES ANNEES 1975-1981}

En observant les données statistiques présentées ci-dessous (graphique 1), on peut constater que la criminalité féminine au Canada dans les années 1975-1981 ne concernait que quelques domaines, y compris les comportements pénalisés ${ }^{16}$. Aux infractions commises par les Canadiennes, il convient tout d'abord d'inclure

11 N. Bourgoin, op. cit., p. 75 et ss.

12 Voir H. Van Gijseghem, Le crime féminin et masculin : deux expressions d'une même délinquence, «Revue Québécoise de Psychologie » 1980, n 1.

13 Voir S. Frigon, L'enfermement des femmes au Canada : une décennie de réformes, « Criminologie » 2002, no spécial 35, DOI : https://doi.org/10.7202/008287ar.

14 E. Comack, S. Brickey, Constituting the Violence of Criminalized Women, "Canadian Journal of Criminology and Criminal Justice" 2007, Vol. 49(1), DOI: https://doi.org/10.3138/5523-48731386-5453, p. 1-36.

15 Ibidem.

16 R. Collette-Carrière, L. Langelier-Biron, op. cit., p. 40-41. 
les crimes contre les biens, les crimes caractérisés par le recours à la violence et d'autres catégories de crimes. Compte tenu du pourcentage dans la réalisation des catégories d'infractions mentionnées par les suspectes, il convient de préciser que les crimes liés aux violations de biens (vols, cambriolages, fraudes) étaient dominants. Au cours de la période de recherche indiquée, ils constituaient la catégorie la plus développée de crimes féminins du point de vue des fréquences. Leur niveau dans les années 1975-1981 a montré une tendance à la hausse (de 65\% à près de $70 \%$ ). Dans le cas de la deuxième catégorie de crimes analysés, à savoir ceux qui ont recours à la violence, on peut avoir l'impression que, pendant la période de recherche indiquée, leur niveau était fondamentalement stable. Dans le contexte des crimes contre les biens, ils constituaient une catégorie qui n'était pas excessivement avancée, en représentant env. $9 \%{ }^{17}$. Les autres crimes indiqués dans le code pénal ne constituaient pas une catégorie trop étendue. Les femmes les ont commis beaucoup plus rarement que les crimes contre les biens. Leur pourcentage dans le nombre total de crimes commis par des femmes variait de $26 \%$ en 1975 à $22 \%$ en 1981 et subissait une légère diminution progressive.

\section{Sélection de catégories de crimes commis par des femmes dans les années 1975 - 1981}

$(\%)$

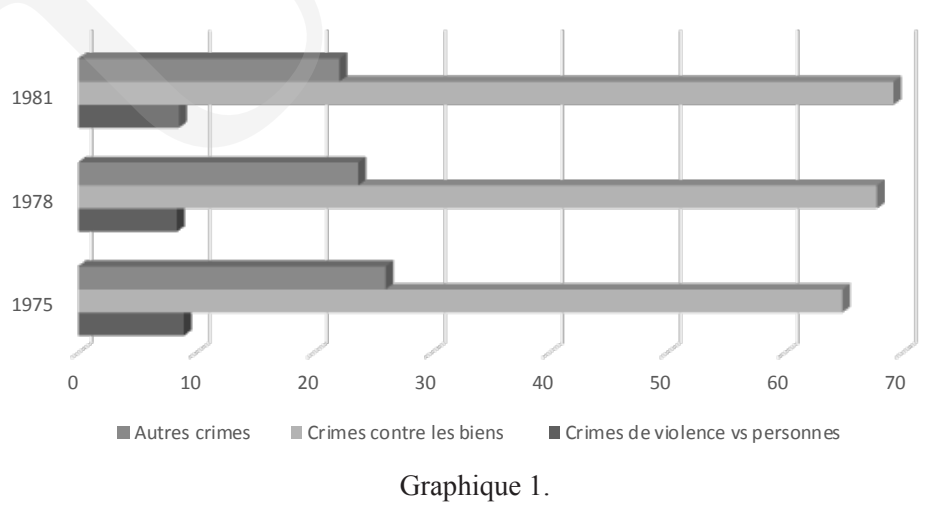

Source : M.A. Bertrand, La femme et le crime, Montréal 1979, p. 57 ; R. Collette-Carrière, L. Langelier-Biron, Du côté des filles et des femmes, leur délinquance, leur criminalité, « Les femmes et la justice pénale »1983, n², DOI : https:// doi.org/10.7202/017179ar, p. 41.

L'analyse du graphique 1 permet de conclure que les infractions dominantes interdites dans l'activité criminogène des Canadiennes dans les années 1975-1981 étaient des crimes contre les biens. Ils constituaient près de $3 / 4$ de tous les crimes commis par les suspectes à cette période-là. Significatif reste le fait que la population

17 Ibidem. 
de femmes entrant en conflit avec la loi ne se soit pas concentrée sur la commission d'infractions de haute nocivité sociale, à savoir de crimes ayant recours à la violence ${ }^{18}$. Ils ont représenté $1 / 10$ de toutes les infractions au cours de la période considérée, en maintenant un niveau modéré et ne montrant aucune tendance à la hausse.

Pour rendre ces considérations encore plus complexes, il a été décidé de présenter ci-après des données plus détaillées sur des catégories spécifiques de crimes commis par le sexe féminin dans les années 1975-1981'19.

Tableau 1. Catégories individuelles de crimes commis par des femmes au Canada (\%)

\begin{tabular}{|l|r|r|r|}
\hline \multicolumn{1}{|c|}{ Sélection des catégories de crimes } & 1975 & 1978 & 1981 \\
\hline Meurtre & 12,3 & 12,1 & 12,4 \\
\hline Crimes sexuels & 1,2 & 0,6 & 1,3 \\
\hline Dommage à la santé & 8,9 & 9,7 & 10,7 \\
\hline Crimes ayant recours à la violence à l'encontre des personnes & 8,0 & 8,6 & 9,5 \\
\hline Vol qualifié & 6,6 & 6,7 & 7,2 \\
\hline Cambriolage & 3,4 & 4,0 & 4,2 \\
\hline Vol de voiture & 3,8 & 4,4 & 5,2 \\
\hline Recel & 10,4 & 12 & 11,8 \\
\hline Fraude & 19,0 & 23,4 & 24,1 \\
\hline Autres crimes contre les biens & 18,0 & 20,2 & 19,7 \\
\hline Crimes contre les jeux et paris & 5,1 & 3,7 & 5,7 \\
\hline Insulte & 9,0 & 10,0 & 10,1 \\
\hline Autres crimes & 10,8 & 10,1 & 10,2 \\
\hline
\end{tabular}

Source : R. Collette-Carrière, L. Langelier-Biron, Du côté des filles et des femmes, leur délinquance, leur criminalité, «Les femmes et la justice pénale » 1983, nº 2, DOI : https://doi.org/10.7202/017179ar, p. 42.

L'analyse des données statistiques de la tableau 1 révèle certaines observations essentielles. En effet, comme déjà indiqué sur la base des données contenues dans le graphique 1, au cours de la période analysée (1975-1981) au Canada, les crimes ayant recours à la violence commis par des femmes ont été maintenus à un niveau stable. Malheureusement, le taux d'homicides (env. 12\%) était légèrement plus élevé dans cette catégorie d'infractions que les autres crimes (les dommages pour la santé ont atteint la fréquence de 9 à 10,7\%, tandis que les autres crimes ayant recours à la violence ont atteint une fréquence de 8 à $9,5 \%$ ), et incidemment a été présentée la fréquence des crimes sexuels commis par les femmes (de 1,2\% à $0,6 \%$ ). Ainsi, même si les crimes ayant recours à la violence n'ont pas été commis trop

18 Cf. plus largement C. Cardi, G. Pruvost, La violence des femmes : un champ de recherche en plein essor, « Le contrôle social des femmes violentes » 2011, nº 8, DOI : https://doi.org/10.4000/ champpenal.8102.

19 R. Collette-Carrière, L. Langelier-Biron, op. cit., p. 42. 
fréquemment, une analyse détaillée permet de conclure que les actes du plus haut degré de nocivité sociale - le meurtre - ont prédominé dans cette catégorie ${ }^{20}$.

Il est intéressant de noter que parmi les crimes contre les biens, dont les Canadiennes ont commis le plus dans les années 1975-1981, la place centrale a été occupée par : le recel et la fraude, qui ont montré une légère tendance à remonter au cours de la période considérée. Celles-ci ont été suivies par des catégories de vols différenciées (par exemple, le cambriolage ou le vol d'une voiture).

Un peu surprenant est le fait que parmi les différentes catégories de crimes susmentionnées, les femmes ont été très souvent auteurs d'injures. Cette infraction représentait 1/10 de toutes les infractions commises par le sexe féminin au cours de la période considérée.

\section{LA CRIMINALITE FEMININE AU CANADA DANS LES ANNEES 1984-1994 ET 1999-2009}

Les périodes de recherche suivantes, qui ont été analysées du point de vue de la criminalité féminine au Canada, étaient deux périodes : 1984-1994 et 1999-2009²1. Cette sélection temporelle du matériel de recherche était principalement dictée par la volonté d'observer la variabilité ou de préserver des tendances criminogènes spécifiques manifestées par les femmes - auteurs. À cette fin, ci-dessous (graphique 2 et 3 ) on a présenté analogiquement les catégories de crimes commis par des suspectes à celles déjà décrites. Comme on peut le voir au graphique 2 , la catégorie prédominante des crimes commis par des Canadiennes dans les années 1984-1994 était toujours celle incluant les infractions contre les biens, ensuite, comme lors de la période de recherche précédente, toutes les autres catégories de crimes, et à la fin (le plus rarement) des crimes ayant recours à la violence contre des tiers. L'analyse préliminaire mène à la conclusion quant à la consolidation des constatations faites dans le domaine de la criminalité chez les Canadiennes sur la base de documents de recherche pour la période de 1975-1981. Cependant, une observation approfondie des données statistiques présentées dans le graphique 2 permet d'indiquer certaines différences significatives en ce qui concerne la population d'auteurs visée. Tout d'abord, il faut noter que même si, dans les années 1984-1994, les infractions contre les biens restaient toujours une catégorie dominante, cependant, le pourcentage de femmes dans leur commission a drastiquement diminué, arrivant à $20 \%$ en 1984. En même temps, le taux de fréquence maximal d'infractions contre les biens commis par des femmes est tombé à près de $25 \%$ en 1993. Ainsi, on peut

${ }^{20}$ Ibidem.

21 T. Hotton Mahony, Les femmes et le système de justice pénale, Canada 2011, www.statcan. gc.ca/pub/89-503-x/2010001/article/11416-fra.pdf [accès : 10.02.2019], p. 23-24. 
voir que la diminution de la fréquence d'exécution des infractions contre les biens dans les années 1984-1994 par rapport à la période 1975-1981 a été importante et a chuté de près de $2 / 3$. Il convient de noter, toutefois, que dans la période d'étude 1984-1994 il y a eu une légère augmentation de la fréquence de cette catégorie de crimes (de $20 \%$ en 1984 à près de $25 \%$ en 1993$)^{22}$.

En observant le graphique 2, on peut constater une légère augmentation de la fréquence des crimes violents contre des tiers parmi les femmes. Leur fréquence dans les années 1975-1981 était de 9 à 10\%. Pendant ce temps, dans la période examinée (1984-1994), on peut indiquer que les femmes poursuivent ces crimes avec une fréquence comprise entre 10 et $12 \%$. Le fait qu'au cours de la période analysée, cette catégorie de crimes a montré une légère tendance croissante, mais lente et régulière, est inquiétant ${ }^{23}$.

Une certaine diminution de la participation des femmes a été marquée dans les années 1984-1994, en ce qui concerne la mise en œuvre d'autres catégories de crimes. Leur fréquence variait de 12 à $17 \%$, ce qui montre une tendance à la hausse similaire à celle des catégories de crimes précédentes. À titre de comparaison, dans les années 1975-1981 les femmes ont commis d'autres crimes avec une fréquence de 22 à $26 \%$ (par conséquent, ils ont représenté $1 / 5$ de tous les crimes dans cette population $)^{24}$.

L'analyse du graphique 2 permet donc de constater que, bien que dans les années 1984-1994 les Canadiennes aient généralement commis moins d'infractions que dans la période d'étude précédente (1975-1981) dans les catégories de crimes examinées on peut observer les tendances à une légère augmentation de la fréquence de leur mise en œuvre (de 2 à $5 \%$ ).

Pourtant, selon le graphique 3 dans les années 1999-2009, les criminologues canadiens ont noté de nouveaux changements dans la structure de la criminalité chez les femmes. L'agencement et la séquence des catégories individuelles d'infractions commises par les auteurs sont restés dans cette période de recherche analogues à ceux indiqués dans les périodes précédentes : 1975-1981 et 1984-1994. Ainsi, les femmes continuaient de commettre des crimes contre les biens, et le plus rarement des crimes violents, mais la fréquence de leurs activités criminogènes a changé.

À partir des données indiquées ci-dessous, il est clair que les crimes contre les biens dominent comme infractions commises par des femmes canadiennes ${ }^{25}$. Dans les années 1999 à 2009, elles ont commis cette catégorie de crimes avec une fréquence allant de 22 à $27 \%$. En comparaison avec les périodes de recherche antérieures, il convient de préciser que cette fréquence était significativement plus

\footnotetext{
22 Ibidem.

${ }^{23}$ Ibidem.

24 R. Collette-Carrière, L. Langelier-Biron, op. cit., p. 41.

25 R. Kong, K. AuCoin, op. cit., p. 11.
} 
Pobrane z czasopisma Studia Iuridica Lublinensia http://studiaiuridica.umcs.pl Data: 26/04/2023 12:55:16

Sélection de catégories de crimes commis par des femmes dans les années $1984-1994(\%)$

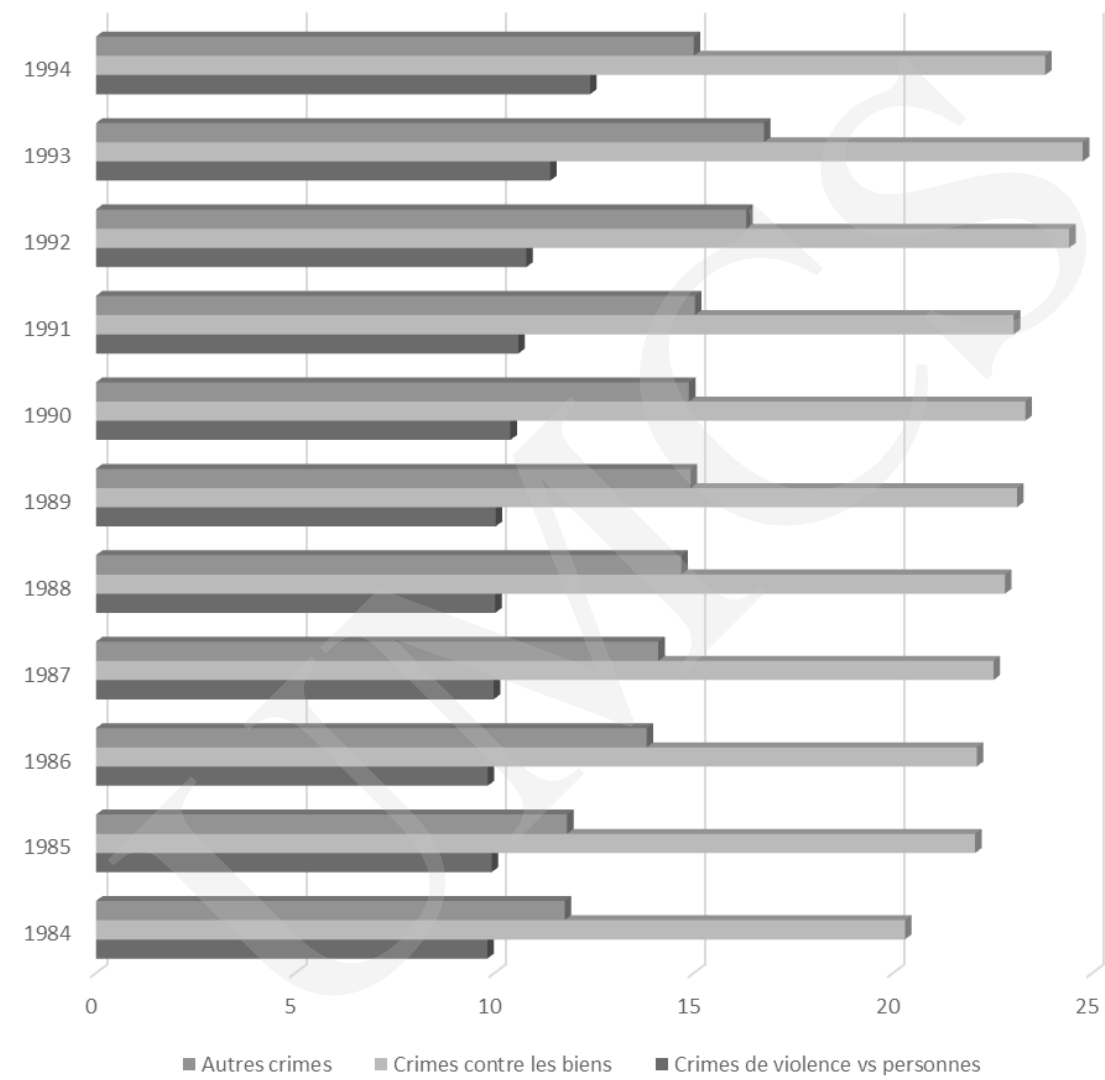

Graphique 2 .

Source : T. Hotton Mahony, Les femmes et le système de justice pénale, Canada 2011, www.statcan.gc.ca/pub/89-503-x/2010001/article/11416-fra.pdf [accès : 10.02.2019], p. 24. Voir aussi : Statistique Canada, Centre canadien de la statistique juridique. Programme de déclaration uniforme de la criminalité agrégée, 1979 à 2009.

faible que dans les années 1975-1981 et légèrement plus élevée (d'env. 2\%) par rapport à la période $1984-1994^{26}$.

Des observations concernant des catégories de crimes impliquant des actes de violence sont cependant inquiétantes ${ }^{27}$. Dans les années 1999-2009, les Canadiennes se sont souvent permis de commettre les infractions en substance. Leur fréquence était de 14,5 à $17 \%$. En comparant les résultats obtenus avec ceux qui se sont produits au cours des périodes de recherche précédentes, il convient de

\footnotetext{
26 T. Hotton Mahony, op. cit., p. 24.

27 R. Kong, K. AuCoin, op. cit., p. 10-11.
} 
Pobrane z czasopisma Studia Iuridica Lublinensia http://studiaiuridica.umes.pl

Data: 26/04/2023 12:55:16

La criminalité féminine au Canada selon les statistiques

constater que, malheureusement, il y a un nombre croissant de crimes impliquant des femmes qui utilisent la violence dans leur cours. Selon les recherches menées dans les années 1975-1981, une femme sur dix était criminellement active dans cette catégorie d'actes criminels, et près de deux femmes sur dix s'engagent actuellement dans la mise en œuvre de ces types de crimes, ce qui reste un phénomène très négatif ${ }^{28}$.

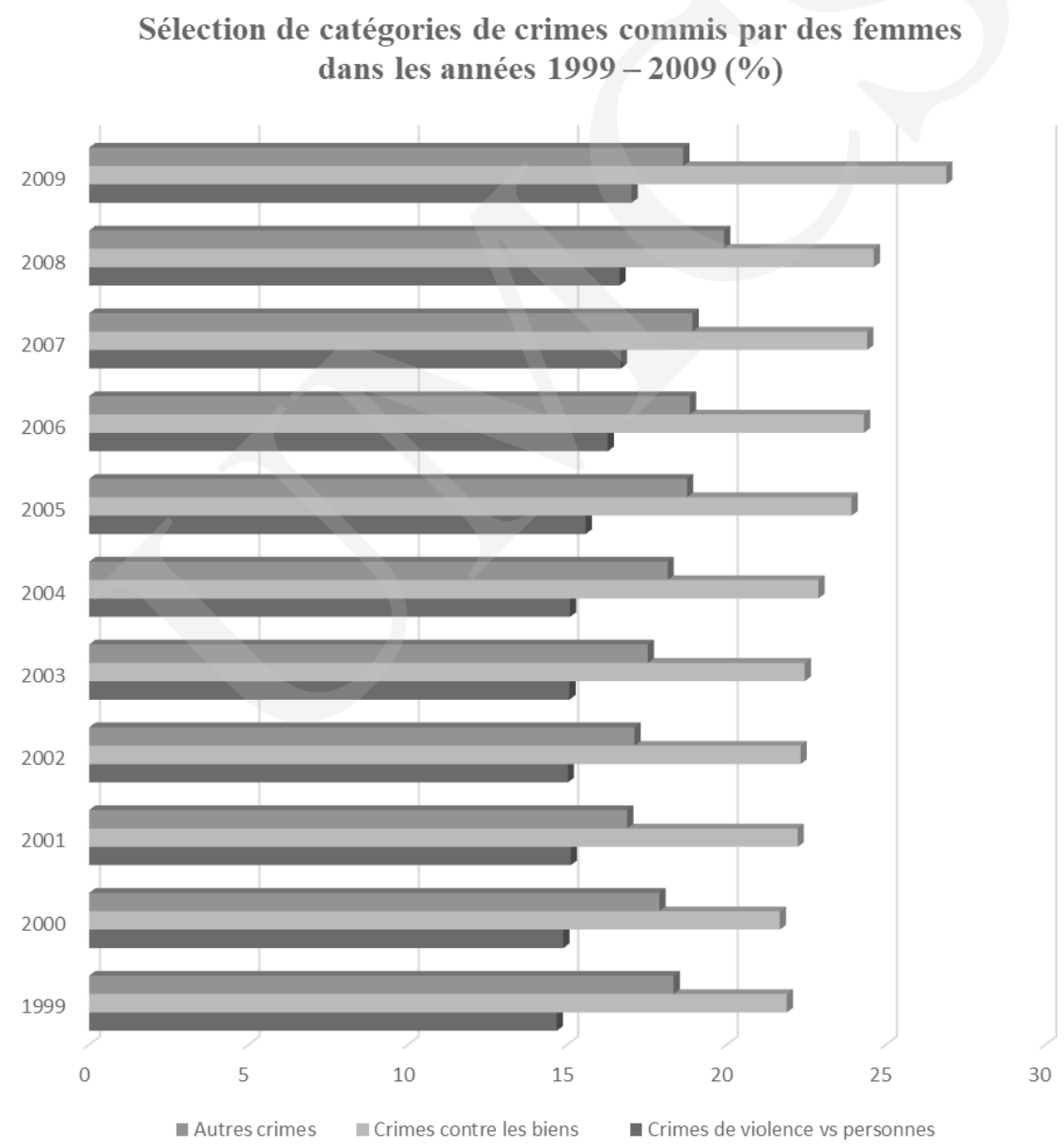

Graphique 3.

Source : T. Hotton Mahony, Les femmes et le système de justice pénale, Canada 2011, www.statcan.gc.ca/pub/89-503-x/2010001/article/11416-fra.pdf [accès : 10.02.2019], p. 24 ; Statistique Canada, Centre canadien de la statistique juridique. Programme de déclaration uniforme de la criminalité agrégée, 1979 à 2009 ; A. Finn, S. Trevethan, G. Carrière, M. Kowalski, Femmes détenues, détenus autochtones et détenus condamnés à perpétuité : un profil instantané d'une journée, « Juristat » 1999, nº 5 .

${ }^{28}$ R. Collette-Carrière, L. Langelier-Biron, op. cit., p. 41. 
En ce qui concerne les catégories d'autres crimes, il est difficile d'indiquer une tendance claire en matière de recherche. Au début, dans les années 1999-2001, leur fréquence a diminué, puis, après 2002, elle a commencé à augmenter. En général, leur fréquence a été au cours de la période examinée (1999-2009) au niveau de 18 à 20\% - plus élevée que dans les années 1984-1994 de près de 5\%.

Pour illustrer mieux ces résultats de la recherche, il a été décidé de présenter les données statistiques suivantes sur l'activité criminelle des contrevenantes Canadiennes dans le domaine de certaines catégories de crimes, qu'elles ont commis en $2009^{29}$. Comme le montre le tableau 2, les femmes ont commis 522 crimes contre les biens, 467 infractions relevant d'autres catégories (dont 107 infractions routières) et 371 actes de violence. Les données statistiques présentées confirment les analyses présentées ci-dessus sur la base du graphique 3 et permettent de formuler des conclusions concrètes.

Dans le cas de crimes contre les biens commis par des femmes, le fraude et le recel continuent de dominer. Les types de vols individuels sont moins souvent commis par les auteurs. De manière significative, parmi les crimes ayant recours à la violence commis en $2009^{30}$, les Canadiennes ont le plus souvent commis des préjudices corporels découlant de lésions de faible gravité, le plus rarement des meurtres et autres crimes ayant des conséquences fatales. Malheureusement, comme auparavant (1984-1994), la fréquence du crime de harcèlement et du recours aux menaces demeure élevée dans la population examinée ${ }^{31}$.

Une nouvelle tendance inquiétante qui peut être observée est la participation relativement avancée des femmes aux deux catégories de crimes commises qui n'apparaissaient pas dans les analyses des périodes de recherche précédentes : les infractions routières et les infractions en matière de drogue. La première d'entre elles apparaît suite à l'accès large des femmes à l'utilisation des véhicules à moteur et résulte de la nécessité de déplacement universel. La seconde concerne l'accès des femmes aux substances intoxicantes et autres substances illégales. Les catégories de crimes mentionnées ci-dessus indiquent la participation active des femmes à la mise en œuvre de crimes qui restent essentiellement bien connus, ce qui indique l'évolution de leurs intérêts criminels. Dans ce contexte, le fait que leur fréquence dans la mise en œuvre des catégories d'infractions indiquées est faible est positif, mais cela ne signifie pas qu'elle n'évoluera pas dans une direction progressive.

\footnotetext{
29 La criminalité chez les femmes..., p. 1.

30 T. Hotton Mahony, op. cit., p. 23-24.

31 Ibidem.
} 
Tableau 2. Sélection de catégories de crimes commis par des femmes au Canada en 2009

\begin{tabular}{|l|c|c|}
\hline \multicolumn{1}{|c|}{ Sélection de catégories de crimes } & $\begin{array}{c}2009 \\
\text { Nombre total }\end{array}$ & $\begin{array}{c}2009 \\
\text { Coefficient par } 100000\end{array}$ \\
\hline Crimes ayant recours à la violence au code pénal & 371,2 & 0,4 \\
\hline Homicide & - & 0,1 \\
\hline Autres infractions causant la mort & - & 1,4 \\
\hline Infractions sexuelles & - & 3,2 \\
\hline Lésion corporelle grave & - & 190,0 \\
\hline Lésion corporelle légère & - & 0,8 \\
\hline Extorsion & - & 18,4 \\
\hline Harcèlement & - & 51,2 \\
\hline Menaces & - & - \\
\hline Crimes contre les biens & 522,0 & 8,4 \\
\hline Vol qualifié & - & 23,1 \\
\hline Cambriolage & - & 13,1 \\
\hline Vol de voiture & - & 43,8 \\
\hline Recel & - & 73,4 \\
\hline Fraude & - & - \\
\hline Autres infractions prévues au code pénal & 359,3 & - \\
\hline Infractions routières prévues au code pénal & 107,4 & 92,6 \\
\hline Infractions en matière de drogue & - & \\
\hline
\end{tabular}

Source : T. Hotton Mahony, Les femmes et le système de justice pénale, Canada 2011, www.statcan.gc.ca/pub/89-503-x/2010001/article/11416-fra.pdf [accès : 10.02.2019], p. 21.

Bien que le meurtre soit rarement commis au Canada ${ }^{32}$, il constitue toujours un crime du plus haut degré de nocivité sociale. En confrontant cette constatation avec l'avis des criminologues canadiens ${ }^{33}$, selon lesquels parmi les contrevenantes les meurtres de proches sont prédominants, on trouvera ci-dessous un résumé statistique visant à vérifier la position présentée ${ }^{34}$.

Comme on peut le voir au tableau 3, dans les années 1997-2009, il y a eu 677 meurtres au Canada, dont les femmes étaient des auteurs ${ }^{35}$. Cela signifie qu'en moyenne par an, les femmes ont privé 52 personnes de leur vie. La liste ci-dessous confirme la thèse selon laquelle les victimes des femmes sont leurs proches ${ }^{36}$. Dans

32 Cf. Statistique Canada, Population, selon l'état matrimonial et le sexe, par province et territoire, Tableau de données du Recensement de 2001, dernière mise à jour : 31 janvier 2007, https:// www150.statcan.gc.ca/t1/tbl1/fr/tv.action?pid=1710001301 [accès : 10.02.2019].

33 Au Canada, le meurtre est un acte prohibé caractérisé comme suit : meurtre au premier degré, meurtre au deuxième degré, homicide involontaire coupable, infanticide. En 2005, il y avait deux victimes de meurtre par 100000 habitants. Voir http://laws-lois.justice.gc.ca/fra/lois/C-46/page-52. html\#h-77 [accès : 10.02.2019].

34 Voir aussi S. Frigon, Les représentations socio-pénales des femmes « maricides » au Canada. 1866-1954, [dans :] C. Bard, F. Chauvaud, M. Perrot, J.-G. Petit (dir.), Femmes et justice pénale, Rennes 2002, p. 209 et ss.

35 Eadem, L'homicide conjugal au féminin d'hier à aujourd' hui, Montréal 2003, p. 22 et ss.

${ }^{36}$ Les relations entre les femmes - meurtrières et leurs partenaires et enfants constituent l'axe des considérations menées dans le contexte historique par S. Frignon. L'auteur analyse les déterminants 
226 cas, il s'agissait d'hommes avec lesquels les suspectes restaient dans des relations intimes (maris, ex-conjoints ou concubins), alors que dans 236 cas - d'autres parents. Il est intéressant de noter que ce sont les parents, et non les enfants des femmes, qui dominaient dans la catégorie des victimes « familiales ». La fréquence des meurtres de personnes connues, qui concernaient 178 personnes, était également assez élevée. Les statistiques ci-dessous confirment donc la tendance générale selon laquelle les femmes qui décident de tuer dans le cercle des victimes se concentrent sur leurs proches. Parmi eux, on peut indiquer les partenaires actuels et ex-partenaires, les parents et les enfants (même si, au Canada, le niveau de cette catégorie de crimes a été très faible $)^{37}$. Plus rarement, elles ont privé de vie leurs connaissances et les meurtres commis par les femmes dont les victimes ont été des inconnus ont été incidents.

Tableau 3. Relations des contrevenantes avec des victimes dans les années 1997-2009

\begin{tabular}{|l|c|c|}
\hline \multicolumn{1}{|c|}{ Lien entre l'auteure présumée et sa victime } & Nombre & $\%$ \\
\hline Homicide & $\mathbf{6 7 7}$ & 100,0 \\
\hline Victime en relation intime avec l'auteur présumée & $\mathbf{2 2 6}$ & 33,4 \\
\hline Conjoint / conjointe & 172 & 0,6 \\
\hline Ex-conjoint / Ex-conjointe & 21 & 9,7 \\
\hline Autre partenaire intime & 33 & 8,6 \\
\hline Autres relations familiales & $\mathbf{2 3 6}$ & 34,9 \\
\hline Mère / père & 159 & 23,5 \\
\hline Fille / fils & 32 & 4,7 \\
\hline Autre membre de la famille & 45 & 6,6 \\
\hline Connaissances & $\mathbf{1 7 8}$ & 26,3 \\
\hline Connaissance dans une relation criminelle & 21 & 3,1 \\
\hline Connaissance & 102 & 15,1 \\
\hline Autre connaissance & 55 & 8,1 \\
\hline Autres & $\mathbf{3 7}$ & 5,5 \\
\hline Etranger & 36 & 5,3 \\
\hline Relation inconnue & 1 & 0,1 \\
\hline
\end{tabular}

Source : T. Hotton Mahony, Les femmes et le système de justice pénale, Canada 2011, www.statcan.gc.ca/pub/89-503-x/2010001/article/11416-fra.pdf [accès : 10.02.2019], p. 23.

Selon les recherches présentées par R. Kong et K. AuCoin dans la population des femmes - contrevenantes, il y avait moins de cas de meurtres commis sous l'influence de l'alcool ou de substances intoxicantes (65\%) que chez les hommes

des meurtres indiqués et le déroulement des procès au Canada dans les années 1866-1954. Cf. eadem, Les représentations..., p. 5 et ss.

37 Seulement 32 meurtres au cours de la période de 13 ans (1997-2009) ont concerné des enfants. Ainsi, en moyenne, les mères ont privé de vie, chaque année, un enfant. Une autre position est présentée par R. Kong et K. AuCoin (op. cit., p. 13). Selon eux, les meurtres d'enfants représentent $28 \%$ du nombre total de meurtres commis par des femmes. 
(plus de 70\%). En outre, une femme sur cinq ayant commis le meurtre présentait des symptômes de maladie mentale (schizophrénie, cyclophrénie et autres troubles psychologiques graves). En cas de meurtre, les femmes ont rarement utilisé des armes à feu, plus souvent elles utilisent un couteau ou donnent des coups directs à la victime. Par conséquent, les victimes de femmes sont poignardées $(43 \%)$ ou battues $(20 \%)^{38}$.

\section{CONCLUSIONS}

En conclusion, il convient de constater que l'analyse du phénomène de la criminalité chez les femmes au Canada présentée dans l'étude portait sur trois périodes de recherche : 1975-1981, 1984-1994 et 1999-2009. La sélection et le nombre d'enquêtes statistiques présentées ont été dictés par le désir de déterminer quelles tendances dominent parmi les activités criminelles des Canadiennes. Comme on peut le voir d'après les cas examinés, les suspectes canadiennes commettent plusieurs catégories spécifiques de crimes qui n'ont pas changé de façon significative au cours des périodes de recherche indiquées. Sans aucun doute, les crimes contre les biens ont dominé parmi eux. Au début, leur fréquence était extrêmement élevée (env. 65-70\% dans les années 1975-1981), pour finalement être réduite à environ 25\% (1999-2009). Les femmes ont volontairement commis deux types de crimes dans cette catégorie : la fraude et le recel, et ont rarement commis différents types de délits de vol. Les criminologues canadiens expliquent la diminution de l'intérêt des femmes pour la criminalité contre les biens en raison de la fréquence accrue d'exécution des infractions de cette catégorie par les hommes. Il convient toutefois de noter que la catégorie des crimes analysés a prévalu parmi les comportements illégaux des suspectes au cours des trois périodes de recherche.

Cependant, on peut observer une sorte d'évolution au niveau des crimes ayant recours à la violence commis par les Canadiennes. Initialement (1975-1981), les infractions indiquées n'avaient pas de fréquence élevée, soit environ $9 \%$ par an. Cependant, au cours des périodes de recherche suivantes, une progression régulière et ascendante de cette catégorie de crimes a été notée. Dans les années 1984-1994, ils étaient au niveau d'environ $11 \%$, alors que dans une autre période de recherche, leur fréquence a été plus élevée, atteignant le niveau de 14,5 à 17\%. Dans ce contexte, il est particulièrement alarmant de constater que parmi les crimes impliquant des actes de violence, on observe également un crime du plus haut degré de nocivité sociale - le meurtre. Les analyses des données statistiques montrent que, selon les points de vue des criminologues, les Canadiennes tendaient à priver de la vie en particulier des personnes parmi leurs proches (partenaires, parents). Dans le contexte

\footnotetext{
38 Ibidem.
} 
des indicateurs mentionnés, le fait que parmi les victimes de meurtres commis par des femmes il y avait relativement rarement leurs enfants (dans la période de 1997-2009 seulement 32 meurtres) est positivement surprenant.

Il convient de noter que parmi les catégories de crimes connues et bien établies parmi les suspectes canadiennes, de nouvelles tendances apparaissent également. Parallèlement au développement économique et culturel, les femmes ont acquis de nouvelles compétences. Certaines d'entre elles impliquent également la possibilité d'entrer dans la voie du conflit avec la loi. L'une d'entre elle est sans aucun doute la conduite d'un véhicule à moteur. Dans le résumé statistique des années 1999-2009 pour la première fois les femmes ont été identifiées comme les auteurs d'infractions de la circulation, mais surtout, leur part dans le nombre total d'infractions n'a pas été élevé. Dans le même temps, on a noté aussi un autre phénomène inquiétant sous la forme de crimes de femmes impliquant des drogues et des substances intoxicantes. Cette tendance est inquiétante car, au cours des périodes de recherche précédentes, la catégorie indiquée des activités criminogènes des femmes n'a pas été divulguée (elles ne concernaient que les hommes). Il semble qu'il est nécessaire donc d'observer l'orientation (dégressivité / progression) des suspectes canadiennes et d'élaborer le plus tôt possible des programmes de prévention efficaces, afin de combattre cette tendance dans une situation où la catégorie divulguée de crimes n'a pas encore atteint un stade avancé de développement.

\section{BIBLIOGRAPHIE}

Bertrand M.A., La femme et le crime, Montréal 1979.

Biel K., Przestępczość dziewcząt. Rodzaje i uwarunkowania, Kraków 2009.

Błachut J., Niektóre koncepcje kryminologiczne a problem przestępczości kobiet, „Archiwum Kryminologii" 1989, t. 16.

Bourgoin N., Les chiffres du crime. Statistiques criminelles et contrôle social (France, 1825-2006), Paris 2008.

Cabalski M., Przemoc stosowana przez kobiety, Kraków 2017.

Cardi C., Pruvost G., La violence des femmes : un champ de recherche en plein essor, « Le contrôle social des femmes violentes » 2011, $\mathrm{n}^{\circ} 8$, DOI : https://doi.org/10.4000/champpenal.8102.

Collectif C., L'Histoire des femmes au Québec depuis quatre siècles, Montréal 1992.

Collette-Carrière R., Langelier-Biron L., Du côté des filles et des femmes, leur délinquance, leur criminalité, «Les femmes et la justice pénale »1983, n² 2, DOI : https://doi.org/10.7202/017179ar.

Comack E., Brickey S., Constituting the Violence of Criminalized Women, "Canadian Journal of Criminology and Criminal Justice" 2007, Vol. 49(1),

DOI: https://doi.org/10.3138/5523-4873-1386-5453.

Dauvergne M., Statistiques sur les tribunaux de juridiction criminelle pour adultes au Canada 2010 2011, 2012, www.statcan.gc.ca/pub/85-002-x/2012001/article/11646-fra.pdf [accès : 10.02.2019].

Dhamen D., Criminalité, femmes et autochtones, http://web.umoncton.ca/umcm-sitesgr/robinsg/ adpu6055h09/edd.pdf [accès : 10.02.2019]. 
Finn A., Trevethan S., Carrière G., Kowalski M., Femmes détenues, détenus autochtones et détenus condamnés à perpétuité: un profil instantané d'une journée, « Juristat»1999, $\mathrm{n}^{\circ} 5$.

Fitzgerald R., Examen des différences entre les sexes quant à la délinquance, « Catalogue de Statistique Canada » 2003, nº 1 .

Frigon S., L'homicide conjugal au féminin d'hier à aujourd' hui, Montréal 2003.

Frigon S., Corps, féminité et dangerosité : de la production de "corps dociles 》 en criminologie, [dans :] S. Frigon, M. Kerisit (dir.), Du corps des femmes : contrôles, surveillances et résistances, Ottawa 2000.

Frigon S., L'enfermement des femmes au Canada : une décennie de réformes, « Criminologie » 2002, $\mathrm{n}^{\mathrm{o}}$ spécial 35, DOI : https://doi.org/10.7202/008287ar.

Frigon S., Les représentations socio-pénales des femmes " maricides 》 au Canada. 1866-1954, [dans :] C. Bard, F. Chauvaud, M. Perrot, J.-G. Petit (dir.), Femmes et justice pénale, Rennes 2002.

Frigon S., Transformations de la philosophie et gestion pénale des femmes justiciables au Canada (1990-2000) : trois cas de figure, « Revue Femmes et Droit » 2006.

Gijseghem H. Van, Le crime féminin et masculin : deux expressions d'une même délinquence, « Revue Québécoise de Psychologie» 1980, $\mathrm{n}^{\circ} 1$.

Grzyb M., Habzda-Siwek E., Pteć a przestepczość. O problemie dysproporcji ptci, „Archiwum Kryminologii” 2013, t. 23.

Hannah-Moffat K., Punishment in Disguise: Penal Governance and Federal Imprisonment of Women in Canada, Toronto 2001.

Hotton Mahony T., Les femmes et le système de justice pénale, Canada 2011, www.statcan.gc.ca/ pub/89-503-x/2010001/article/11416-fra.pdf [accès : 10.02.2019].

http://laws-lois.justice.gc.ca/fra/lois/C-46/page-52.html\#h-77 [accès : 10.02.2019].

Kong R., AuCoin K., Les contrevenantes au Canada, « Juristat» 2008, $\mathrm{n}^{\circ} 1$.

La criminalité chez les femmes au Québec et au Canada, www.alterjustice.org/u9/criminalite_femmes. $\mathrm{html}$ [accès : 10.02.2019].

Majchrzyk Z., Kiedy kobieta zabija, Warszawa 2009.

Myles B., Rapport de Statistique Canada - Les crimes avec violence en hausse chez les femmes, 2008, www.ledevoir.com/societe/175671/rapport-de-statistique-canada-les-crimes-avec-violence-enhausse-chez-les-femmes [accès : 10.02.2019].

Statistique Canada, Centre canadien de la statistique juridique. Programme de déclaration uniforme de la criminalité agrégée, 1979 à 2009.

Statistique Canada, Population, selon l'état matrimonial et le sexe, par province et territoire, Tableau de données du Recensement de 2001, dernière mise à jour : 31 janvier 2007, https://www150. statcan.gc.ca/t1/tbl1/fr/tv.action?pid=1710001301 [accès : 10.02.2019].

Wallace M., Turner J., Matarazzo A., Babyak C., La mesure de la criminalité au Canada : présentation de l'indice de gravité de la criminalité et des améliorations au Programme de déclaration uniforme de la criminalité, Ottawa 2009.

\section{STRESZCZENIE}

Celem niniejszego opracowania była charakterystyka zjawiska przestępczości kobiecej w Kanadzie z perspektywy analizy danych statystycznych. W związku z tym przedstawiono zestawienia uwzględniające częstotliwość badanego zjawiska w trzech okresach badawczych: 1975-1981, 1984-1994 oraz 1999-2009. Ustalono, że kanadyjskie sprawczynie realizują utrwalone kategorie przestępstw (przestępstwa przeciwko mieniu, przestępstwa z użyciem przemocy), których częstotliwość ulegała zmianie w badanych okresach. W kontekście przeprowadzonych badań niepokojące jest zwłaszcza zjawisko wzrostu częstotliwości czynów zabronionych kobiet, dokonywanych z użyciem 
Pobrane z czasopisma Studia Iuridica Lublinensia http://studiaiuridica.umes.pl Data: 26/04/2023 12:55:16

przemocy. Fakt ten świadczy o wzroście patologizacji ich zachowań. Ponadto w ostatnim z okresów badawczych (1999-2009) odnotowano nowe tendencje w zakresie kryminogennej aktywności kobiet - przestępstwa drogowe oraz przestępstwa dokonane z udziałem narkotyków i środków odurzających, jednak ich poziom nie był wysoki.

Słowa kluczowe: przestępczość kobieca; kryminogenna aktywność kobiet w Kanadzie

\section{SUMMARY}

The aim of this paper was to describe the phenomenon of woman's crime in Canada from the perspective of statistical data analysis. For this reason, the statements regarding the frequency of the studied phenomenon in three research periods were presented: 1975-1981, 1984-1994 and 1999-2009. The Canadian woman perpetrators were found to have established categories of crimes (property crimes, violent crimes), the frequency of which has changed during the periods under investigation. In the context of the studies carried out, in particular, the phenomenon of the increase in the frequency of woman's offences committed using violence, remains a matter of concern. This fact indicates an increase in the pathologization of their behaviours. Moreover, in the last research period (1999-2009) there were also new trends in the criminal activity of women - road traffic offences and offences involving drugs and narcotic drugs, however, their level was not high.

Keywords: woman's crime; categories of woman's crime in Canada 